\title{
Physical Parameter Estimation of Linear Voltage Regulators using Model-based Approach
}

\author{
Ng Len Luet ${ }^{1}$, Mohd Hairi Mohd Zaman ${ }^{2}$, Asraf Mohamed Moubark ${ }^{3}$, M Marzuki Mustafa ${ }^{4}$ \\ Department of Electrical, Electronic and Systems Engineering \\ Faculty of Engineering and Built Environment, Universiti Kebangsaan Malaysia \\ 43600 Bangi, Selangor, Malaysia
}

\begin{abstract}
Electronic systems are becoming increasingly sophisticated due to the emergence of advanced technology, which can produce robust integrated circuits by reducing the dimensions of transistors to just a few nanometers. Furthermore, most electronic systems nowadays are in the form of system-on-chip and thus require stable voltage specifications. One of the critical electronic components is the linear voltage regulator (LVR). LVRs are types of power converter used to maintain a stable and constant DC voltage to the load. Therefore, LVR stability is an essential aspect of voltage regulator design. The main factor influencing the stability of LVRs is the load disturbance. In general, disturbances such as a sudden change in load current can be compensated for by an output capacitor, which, contains a parasitic element known as equivalent series resistance (ESR). Therefore, the ESR and output capacitor specified in the datasheet is essential to compensate for load disturbance. However, LVR manufacturers typically do not provide detailed information, such as the internal physical parameters associated with the LVR in the datasheet. This situation leads to difficulties in identifying the behavior and stability of LVR. Therefore, this study aims to develop a method for estimating the internal physical parameters of LVR circuits that are difficult to measure directly by using a model-based approach (MBA). In this study, the MBA estimates the LVR model transfer function by analyzing the input and output signals via a linear regression method. Simulations through MATLAB and OrCAD Capture CIS software verify the estimated LVR model transfer function. Results show that the MBA has an excellent performance in estimating the physical parameters of LVRs and determining their stability.
\end{abstract}

Keywords-Linear voltage regulator; stability; capacitor; equivalent series resistance; physical parameter; model-based approach

\section{INTRODUCTION}

Smart electronic devices or system-on-chip (SoC) applications have become an integral part of our daily necessities. This situation is due to the rapid development of technology that affects the way of life of individuals in the aspects of communication, learning, and thinking [1]. This development follows Moore's Law, which states that the number of transistors doubles every two years by reducing the dimensions of its transistors. With millions of transistors, stable voltage specifications are crucial in such SoC applications. Therefore, linear voltage regulators (LVRs) play an essential role in the field.

LVRs are a power converters that stabilize the output voltage within an acceptable range. Generally, an unstable output voltage for electronic devices causes oscillatory transients, harmonics distortion and noise. All of these effects degrade the performance of electronic devices. Therefore, LVRs must be able to maintain a steady output voltage and be unaffected by changes in conditions [2].

In general, the main factor influencing the stability of LVRs is the disturbance, including a sudden change in load current. This disturbance can usually be compensated for by an output capacitor, which acts as an energy storage element. However, the output capacitor consists of a parasitic element called equivalent series resistance (ESR), which plays a critical role in the compensation of disturbance [3], [4]. Generally, a high ESR leads to large undershoot in transient response, and thus generates an unstable output voltage. Although a low ESR is always encouraged, it is not always the best option. When the ESR is too low, oscillation occurs and finally destroys the stability of the LVR. Hence, an optimum low ESR is encouraged in LVR design [5], [6], [7], [8].

Although LVR manufacturers provide the ESR and output capacitance information, they do not specify in the datasheet the required stability information, such as the internal physical parameters of the circuit. Hence, engineers face problems in analyzing and achieving stability for the LVRs they use. The available method for analyzing the stability of LVRs involves measuring the transient response manually via a real LVR circuit [9], [10], [11]. However, the process is time-consuming and limits the manufacturing productivity rate. Data-driven methods have been developed to analyze the failure regions of LVRs [12], [13], but they do not involve any circuit modeling.

Besides, some researches have been conducted to analyze the electronic device failure based on circuit models. For instance, the impact on the variation of the circuit transfer function coefficients has been investigated in detail to measure its performance [14], [15], [16], [17]. Furthermore, much research has been conducted to model the power management components, especially for the switching type power converters [18], [19], [20], [21], [22], [23]. However, most works, either modelbased or data-driven method, assume the circuit under test as a black box system and did not consider the partially known physical parameters that exist in the circuit. For instance, in the LVR circuit, the output capacitor and feedback resistors can be regarded as the partially known parameters. Therefore, the current work aims to develop a model-based approach (MBA) to estimate the physical parameters of LVRs adequately via the linear regression (LR) method and subsequently determine the LVR stability by analyzing the transient response. 


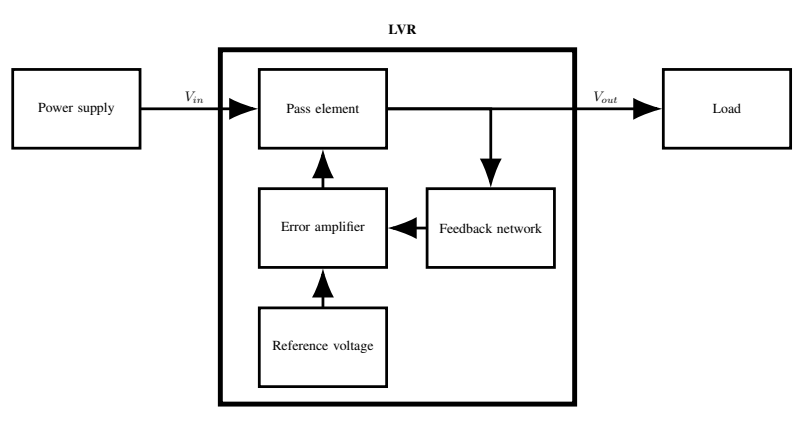

Fig. 1. Block Diagram of LVR.

\section{A. Linear Voltage Regulator}

LVR consists of four main components, namely, reference voltage, error amplifier, feedback circuit, and pass element. Fig. 1 depicts the basic block diagram of an LVR. The error amplifier detects the output voltage through the feedback network and compares it with the reference voltage, thereby responding to the pass element. The pass element can be a BJT or MOSFET, and it maintains the voltage level such that the output voltage has the same value as the reference voltage by controlling the flow of current. For example, when the output voltage decreases due to a sudden change in load, the error amplifier triggers the pass element to control the current flow from the power supply to the load to ensure that the output voltage is at the same level as the reference voltage. The output voltage rises again to the initially controlled voltage [24].

\section{B. Output Capacitor}

A capacitor is a type of passive component that contains two terminals to store electrostatic energy in the electric field. Typically, a capacitor connected in parallel to a circuit output is known as the output capacitor. The output capacitor acts as energy storage. The electrical energy stored is utilized during a sudden change in load. This component minimizes the effect of the disturbance by decreasing the ripple on the output voltage. Therefore, the output capacitor is a critical element in the stability aspect of LVRs.

In general, the value of the output capacitor exerts a significant impact on the stability of LVRs because the output capacitor influences the damping of the output voltage during the transient response. Low capacitor values are generally preferred. However, if the overall impedance of the output capacitor is too low, then oscillation or ring effect occurs and eventually reduces the stability of the output voltage.

\section{Equivalent Series Resistance}

Ideally, capacitors are considered as pure elements and contain only capacitance. However, practically, capacitors used in reality are not pure elements and contain impurity. This impurity contributes to the existence of ESR. Typically, a resistor connected in series with the output capacitor simulates the ESR, as depicted in Fig. 2.

ESR values are essential in achieving LVR stability as they reflect characteristics that are essential to the output voltage, such as oscillation and undershoot during the transient response. For example, when the ESR is too high, it causes the

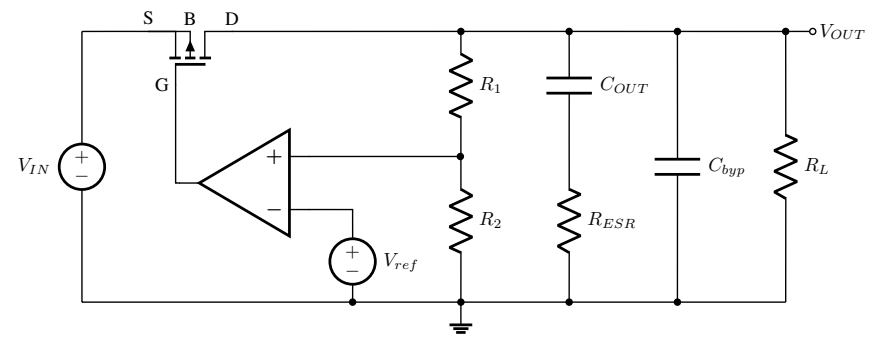

Fig. 2. ESR Connected in Series with the Output Capacitor.

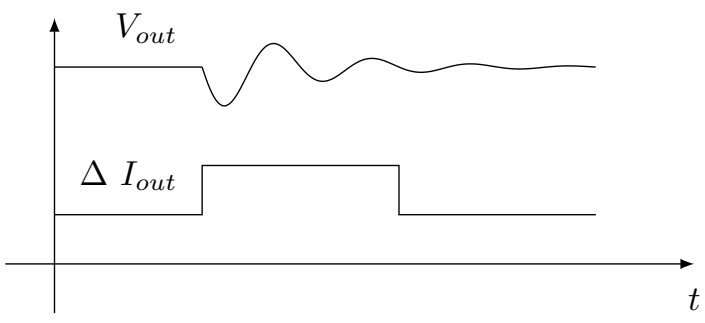

Fig. 3. Unstable Transient Response with Low ESR.

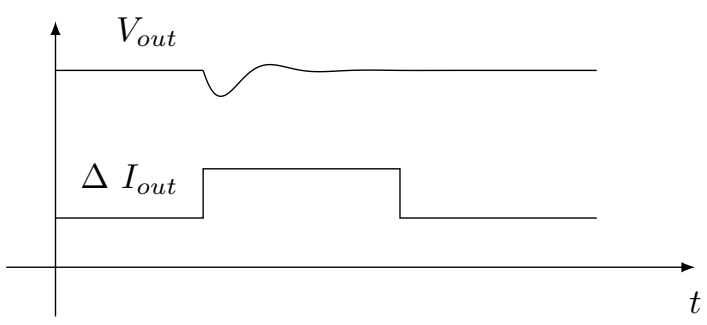

Fig. 4. Stable Transient Response with Optimum ESR.

output voltage to be less than the minimum operating voltage. Finally, this condition contributes to the unstable operation of the LVR. The difference between the output voltage and the minimum operating voltage is known as the undershoot. In sum, the higher the ESR is, the more significant the undershoot will be. This condition contributes to the instability of LVRs.

\section{Linear Voltage Regulator Stability}

The main factor influencing the stability of LVRs is the load disturbance. For example, disturbances, such as sudden changes in load current due to short circuits, have a significant impact on LVR stability. Therefore, the designed LVR must be able to maintain a stable output voltage even if the current change occurs in a short period [12]. Fig. 3 and 4 show two examples of the relationship between ESR and transient response.

Fig. 3 shows that oscillations occur after the first peak, which indicates that the output voltage is unstable. Meanwhile, Fig. 4 depicts a stable output voltage. On the basis of both figures that illustrate the relationship between ESR and output voltage, we conclude that an extremely low ESR can cause oscillations and finally generate an unstable output voltage. Furthermore, LVR stability can be determined by the number of oscillations. For a stable LVR, in practice, the number of oscillations should be less than four. 


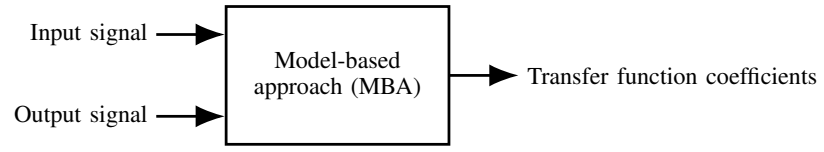

Fig. 5. Block Diagram of MBA Process.

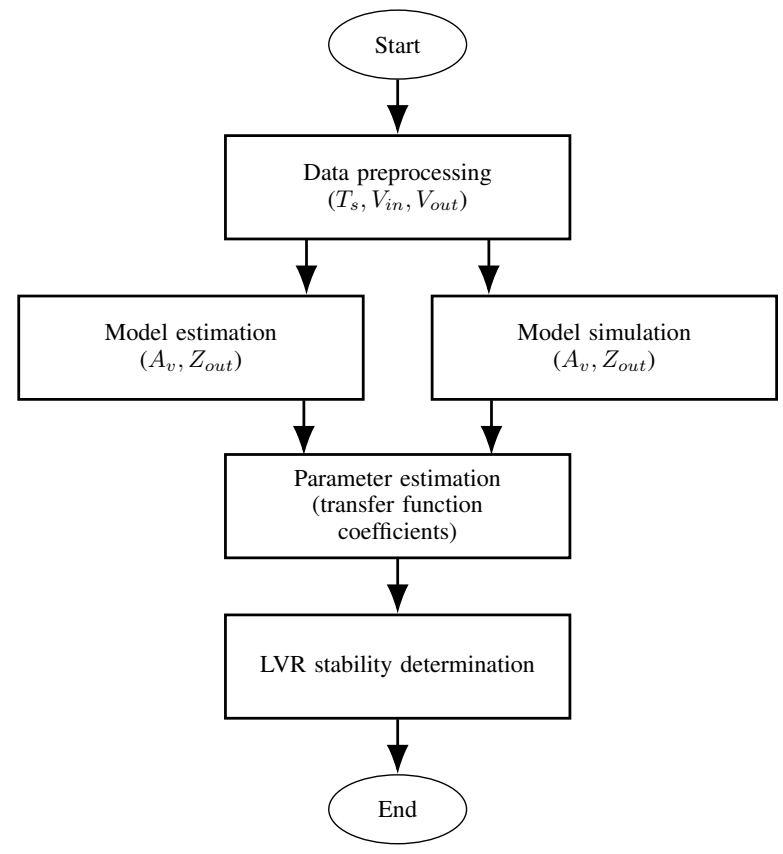

Fig. 6. Overall Flowchart of MBA Process.

\section{METHODS}

\section{A. Model-based Approach}

An MBA is a method for describing system behavior in the forms of transfer functions by analyzing the circuit. The transfer function of a system provides useful information regarding the input and output, such as stability, steady-state response, and dynamic response [25], [26]. In this study, the MBA is aimed at identifying all the coefficients or unknown parameters presented in a transfer function through the analysis of input and output signals. Fig. 5 depicts a simplified block diagram of the MBA process.

The MBA in this study utilizes the LR method to estimate the transfer function. LR is a method for modeling the relationship between two or more variables in a linear equation until they reach the desired level. This study aims to develop an efficient method for estimating the internal physical parameters of the LVR circuit on the basis of the LR method before determining the LVR stability. The study involves five main steps: preprocessing of data, model estimation, model simulation, parameter estimation, and determination of LVR stability. Fig. 6 displays the flowchart of this study.

This process starts with the preprocessing of data, which is aimed at determining the type of input signal. The model estimation process aims to obtain the transfer functions of output impedance, $Z_{\text {out }}$ and the voltage gain, $A_{v}$ via circuit analysis. At the same time, model simulations in the form of $Z_{\text {out }}$ and $A_{v}$ are carried out using OrCAD Capture CIS software. By

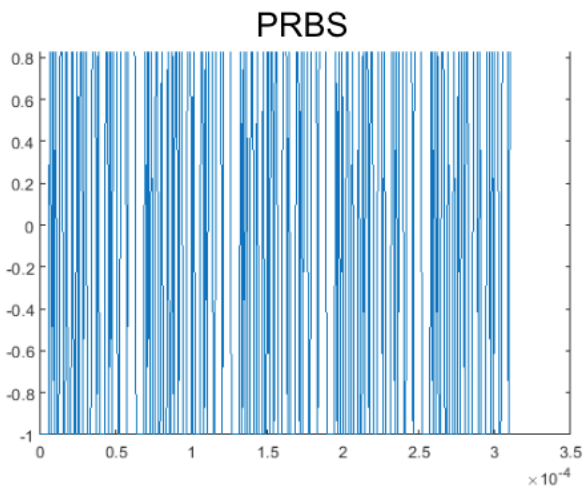

Fig. 7. PRBS Signal.

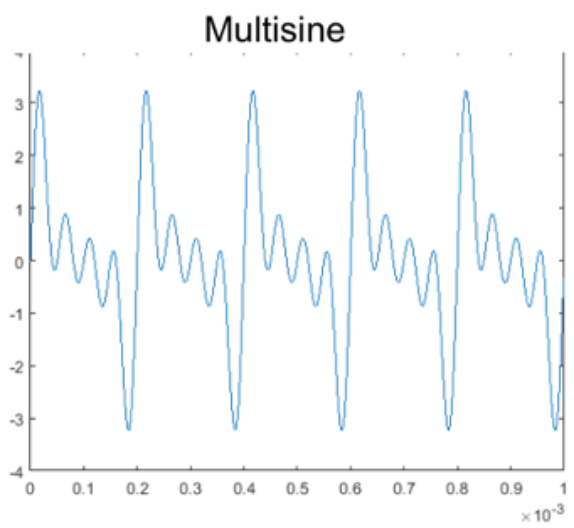

Fig. 8. Multisine Signal.

comparing $A_{v}$ from model estimation and that from model simulation, we obtain the estimated physical parameters in this process. Finally, LVR stability is determined by using the estimated $Z_{\text {out }}$ with estimated physical parameters.

\section{B. Preprocessing Data}

The preprocessing of the data is intended to determine the type of excitation signal or input signal to excite the tested LVR circuit before the model estimation process is carried out. Two input signals are investigated: (a) PRBS signal and (b) multisine signal. The purpose of studying both signals is to select the input signal that is most appropriate for this study to increase the accuracy of the estimation of physical parameters.

The PRBS signal is known as a pseudorandom binary sequence. It is a succession of rectangular pulses that display statistical behavior similar to a random sequence. The PRBS signal is also a perfect white noise that is often used as a test signal. Fig. 7 shows an example of the PRBS signal.

The multisine signal is known as a multifrequency sinusoidal signal. It is an addition of at least two sinusoidal signals with different frequencies. Multisine is usually used to excite the tested circuit by manipulating the frequency of each sinusoidal signal. Fig. 8 shows an example of a multisine signal. 


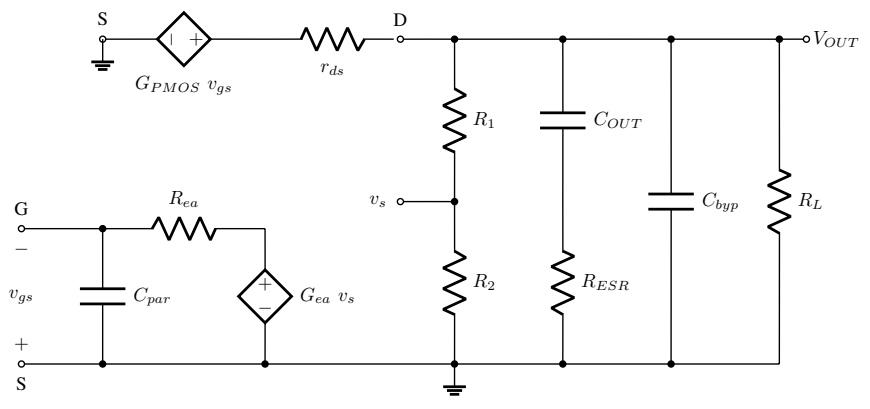

Fig. 9. LVR Circuit for $Z_{\text {out }}$ Model.

Theoretically, both signals are appropriate for this study. However, practically, PRBS is challenging to generate in the OrCAD Capture CIS software because this software does not supply PRBS generator components. Hence, the signal should be built from scratch by using discrete components. Thus, the multisine signal is selected as the input signal of the LVR circuit.

\section{Model Estimation}

Once the input signal is specified, the subsequent model estimation can proceed. Generally, the LVR model estimation aims to obtain the model or transfer function of output impedance, $Z_{\text {out }}$ and voltage gain, $A_{v}$ through circuit analysis. Output impedance, $Z_{\text {out }}$ describes the relationship between the output voltage and the output current during the transient response; it is defined as

$$
Z_{\text {out }}(s)=\frac{v_{\text {out }}(s)}{i_{\text {out }}(s)}
$$

$Z_{\text {out }}$ model is derived on the basis of (1) and the circuit analysis of the output impedance model, as shown in Fig. 9. Small-signal analysis on the LVR circuit, as shown in Fig. 9, is carried out to detect the effect of a sudden change in load current to an output voltage.

$Z_{\text {out }}$ in the discrete time domain can then be defined as

$$
Z_{\text {out }_{C L}}(z)=\frac{1}{Z_{1}(z) Z_{2}(z)}
$$

where

$$
\begin{aligned}
Z_{1}(z)= & \frac{2}{T_{s}}\left(\frac{1-z^{-1}}{1+z^{-1}}\right) C_{b}+\frac{1}{R_{1}+R_{2}}+\frac{1}{R_{L}} \\
& +\frac{1}{r_{d s}}+\frac{1}{\left(\frac{\frac{2}{T_{s}}\left(\frac{1-z^{-1}}{1+z^{-1}}\right) C_{\text {out }}}{(}+R_{\text {esr }}\right)}
\end{aligned}
$$

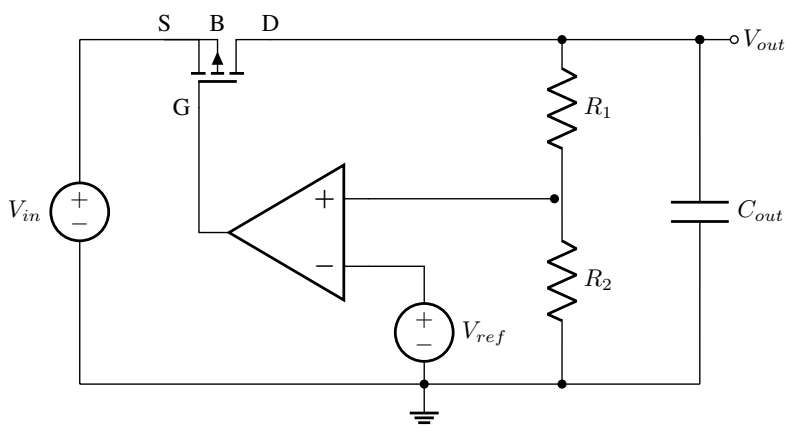

Fig. 10. LVR Circuit for $A_{v}$ Model.

$$
\begin{aligned}
Z_{2}(z)=1 & +G_{f b} G_{e a} g_{m} r_{d s}\left(\frac{Z_{3}(z)}{r_{d s}+Z_{3}(z)}\right) \\
& \times\left(\frac{1}{1+\frac{2}{T_{s}}\left(\frac{1-z^{-1}}{1+z^{-1}}\right) C_{g s} R_{o a}}\right)
\end{aligned}
$$

$$
\begin{aligned}
Z_{3}(z)= & \left(\frac{2}{T_{s}}\left(\frac{1-z^{-1}}{1+z^{-1}}\right) C_{b}+\frac{1}{R_{L}}\right) \\
& +\left(\frac{1}{R_{\text {esr }}+\frac{2}{\frac{2}{T_{S}}\left(\frac{1-z^{-1}}{1+z^{-1}}\right) C_{\text {out }}}}\right)
\end{aligned}
$$

and

$$
G_{f b}=\frac{R_{2}}{R_{2}+R_{1}}
$$

Next, the same method is used to obtain the transfer function of the $A_{v}$ model but with different equations and circuit. $A_{v}$ is derived as

$$
A_{v}(s)=\frac{v_{\text {out }}(s)}{v_{\text {in }}(s)}
$$

and the circuit analysis is conducted on the basis of the voltage gain model, as shown in Fig. 10.

Finally, the transfer function of $A_{v}(z)$ in the discrete-time domain is obtained on the basis of (8).

$$
A_{v}(z)=\frac{1+g_{m} r_{d s}}{1+Z_{4}(z)+Z_{5}(z)}
$$

where

$$
Z_{4}(z)=\frac{2}{T_{s}}\left(\frac{1-z^{-1}}{1+z^{-1}}\right) C_{\text {out }} r_{d s}+\frac{r_{d s}}{R_{1}+R_{2}}
$$




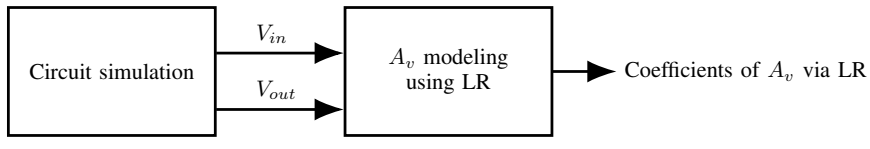

Fig. 11. Block Diagram of the MBA Process.

$$
Z_{5}(z)=\frac{g_{m} r_{d s}}{\left(R_{1}+R_{2}\right)\left(1+\frac{2}{T_{s}}\left(\frac{1-z^{-1}}{1+z^{-1}}\right) C_{g s} R_{o a}\right)}
$$

In general, (8) can be simplified and expressed as

$$
A_{v}(z)=\frac{b_{0}+b_{1} z^{-1}+b_{2} z^{-2}}{a_{0}+a_{1} z^{-1}+a_{2} z^{-2}}
$$

where $b_{0}$ to $a_{2}$ are given as

$$
\begin{gathered}
b_{0}=N_{1} C_{g s} R_{o a}+N_{2} C_{g s} R_{o a} g_{m}+N_{3} g_{m}+N_{4} \\
b_{1}=N_{5} g_{m}+N_{6}
\end{gathered}
$$

$b_{2}=N_{7} C_{g s} R_{o a}+N_{8} C_{g s} R_{o a} g_{m}+N_{9} g_{m}+N_{10}$

$$
a_{0}=D_{1} C_{g s} R_{o a}+D_{2} G_{e a} g_{m}+D_{3}
$$

$$
a_{1}=D_{4} C_{g s} R_{o a}+D_{5} G_{e a} g_{m}+D_{6}
$$

$$
a_{2}=D_{7} C_{g s} R_{o a}+D_{8} G_{e a} g_{m}+D_{9}
$$

where $N_{1}$ to $N_{10}$ represents the known constant values of the numerator while $D_{1}$ to $D_{9}$ represent the known constant values of the denominator that is made up of known and measurable physical parameters, such as $R_{1}, R_{2}, C_{\text {out }}$, and $r_{d s}$. Equations (12) to (17) show that the following physical parameters need to be estimated: $C_{g s} R_{o a}, C_{g s} R_{o a} g_{m}, g_{m}$, and $G_{e a} g_{m}$. To decrease the number of coefficients in the transfer function of $A_{v}(z)$, we can divide the result of (11) by $a_{0}$. Finally, the simplified transfer function can be written as (18).

$$
A_{v_{M A L}}(z)=\frac{b_{0_{M A L}}+b_{1_{M A L}} z^{-1}+b_{2_{M A L}} z^{-2}}{1+a_{1_{M A L}} z^{-1}+a_{2_{M A L}} z^{-2}}
$$

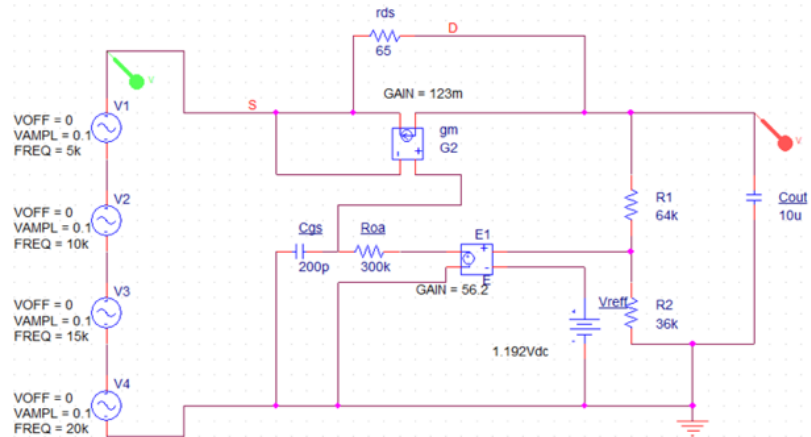

Fig. 12. Circuit Simulation of $A_{v}$ Model.

\section{Model Simulation}

The next process in the MBA is the model simulation of the LVR circuit. This process aims to obtain the coefficients of the $A_{v}$ model through the LR method by analyzing the input and output signals. Fig. 11 depicts the block diagram of the model simulation process.

Circuit simulation is conducted in OrCAD Capture CIS software. This process is aimed at obtaining the input signal, $v_{\text {in }}$ and output signal, $v_{\text {out }}$ of the $A_{v}$ model. The circuit simulation for the $A_{v}$ model, in the small-signal analysis circuit form, is constructed and shown in Fig. 12.

In this study, the selected input signal is a multisine signal with four different frequencies, namely, 5, 10, 15, and 20 $\mathrm{kHz}$. Generally, each sinusoidal signal is capable of testing two frequency components at a time. Therefore, at least three different types of sinusoidal signals are needed for six frequency components or coefficients, as in (11). However, four sinusoidal signals are used to improve the accuracy of the estimated model.

Next, the input and output signals obtained from the LVR circuit simulation are used for the $A_{v}$ modeling of the LVR circuit based on the LR method with the aid of MATLAB software. As the $A_{v}$ model with the LR method generated by MATLAB is derived as

$$
A_{v_{L R}}(z)=\frac{y(k)}{x(k)}=\frac{b_{0_{L R}}+b_{1_{L R}} z^{-1}+b_{2_{L R}} z^{-2}}{1+a_{1_{L R}} z^{-1}+a_{2_{L R}} z^{-2}}
$$

then (19) needs to be converted into

$$
\begin{aligned}
y(k)= & -a_{1_{L R}} y(k-1)-a_{2_{L R}} y(k-2) \\
& +b_{0_{L R}} x(k)+b_{1_{L R}} x(k-1) \\
& +b_{2_{L R}} x(k-2)
\end{aligned}
$$

Then, $\beta$ is defined as

$$
\begin{aligned}
\beta & =\left[\beta_{0}, \beta_{1}, \beta_{2}, \beta_{3}, \beta_{4}, \beta_{5}\right]^{T} \\
& =\left[-a_{1_{L R}},-a_{2_{L R}}, b_{0_{L R}}, b_{1_{L R}}, b_{2_{L R}}\right]^{T}
\end{aligned}
$$




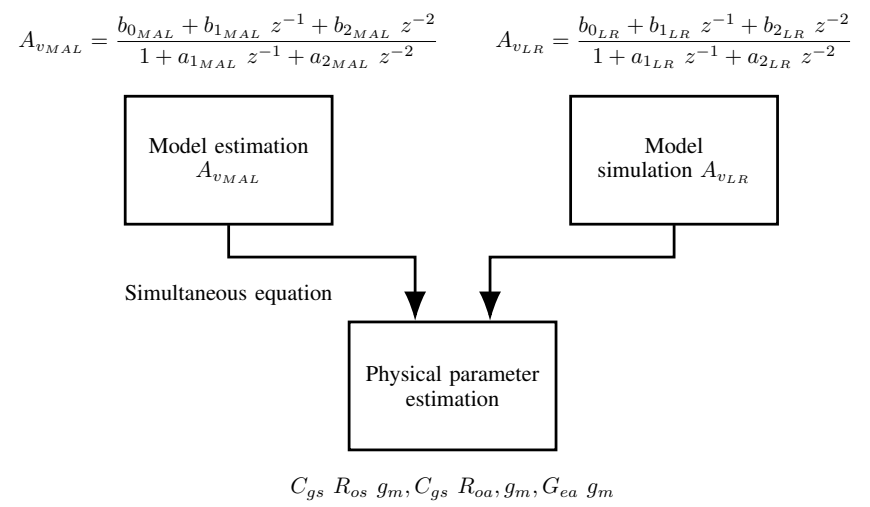

Fig. 13. Block Diagram of the Process of Parameter Estimation.

Equation (21) is used to determine the polarity of the estimated parameters. Furthermore, the derivation of vector $\beta$ is started by the general equation of the LR method and is defined as

$$
\begin{gathered}
y=x^{T} \beta \\
\beta=\left(x^{T} x\right)^{-1} x^{T} y
\end{gathered}
$$

where $y$ is the output signal and $x$ is the input signal. Finally, (21) is used to determine the polarity of the estimated parameters while (23) is used to estimate the coefficients such as $b_{0_{L R}}, b_{1_{L R}}, b_{2_{L R}}, a_{1_{L R}}$, and $a_{2_{L R}}$ in the $A_{v_{L R}}$ model. The estimated coefficients are used to estimate the physical parameters of the LVR circuit in the next process.

\section{E. Physical Parameter Estimation}

This section explains the process of physical parameter estimation by comparing the two voltage gain transfer functions obtained from the model simulation, $A_{v_{L R}}$, and the model estimation, $A_{v_{M A L}}$. This process aims to estimate the physical parameters that are difficult to measure, i.e., $C_{g s} R_{o a}$, $C_{g s} R_{o a} g_{m}, g_{m}$, and $G_{e a} g_{m}$, by using the simultaneous equation solution approach. Fig. 13 depicts the block diagram of the process of parameter estimation.

The coefficients of $A_{v_{L R}}$ and $A_{v_{M A L}}$ are equated to solve the following equations simultaneously:

$$
\begin{aligned}
& b_{0_{M A L}}=b_{0_{L R}} \\
& b_{1_{M A L}}=b_{1_{L R}} \\
& b_{2_{M A L}}=b_{2_{L R}} \\
& a_{1_{M A L}}=a_{1_{L R}} \\
& a_{2_{M A L}}=a_{2_{L R}}
\end{aligned}
$$

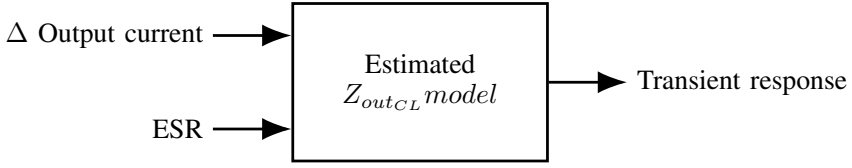

Fig. 14. Block Diagram of the Process of Obtaining a Transient Response via the Estimated Model.

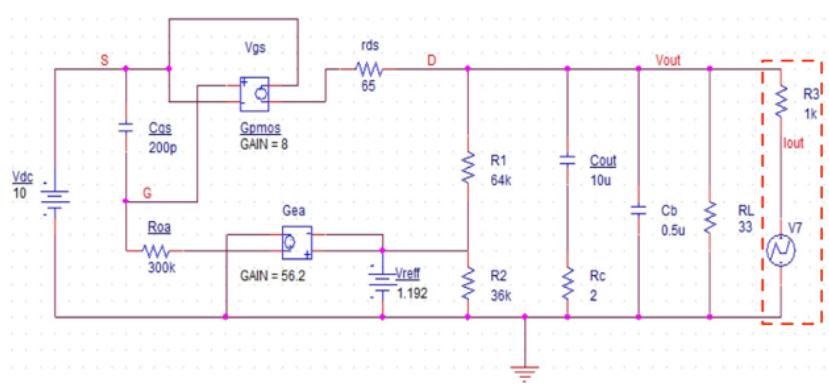

Fig. 15. LVR Circuit Simulation of $Z_{\text {out }}(z)$ Model.

Then, the physical parameters, i.e., $C_{g s} R_{o a}, C_{g s} R_{o a} g_{m}$, $g_{m}$, and $G_{e a} g_{m}$, are estimated. Thereafter, these estimated physical parameters are substituted into the $Z_{\text {out }}(z)$ model, as shown in (2). Moreover, parameters that are easy to measure and obtain, such as $R_{1}, R_{2}, C_{\text {out }}$, and $r_{d s}$, are also substituted by their measured values in (2). Finally, a complete estimated $Z_{\text {out }_{C L}}(z)$ is obtained.

\section{F. Determination of LVR Stability}

This section explains the process of determination of LVR stability by analyzing the transient response of the LVR. The transient response is obtained when the input, such as a sudden change in the output current and ESR values, is applied to the estimated $Z_{\text {out }}$ CL $(z)$ model. Fig. 14 depicts the process of obtaining a transient response.

The circuit simulation, as shown in Fig. 15, is used to generate the output voltage during the transient response. This result is known as the $Z_{\text {out }_{C L}}(z)$ simulation result. Furthermore, instead of directly analyzing the output voltage from the simulation, we can generate the output voltage via the estimated model, the process of which is shown in Fig. 14. A disturbance signal, such as a sudden change in the output current at the tested ESR value, is applied to the circuit. Sudden change conditions can be simulated using a resistor, $R_{3}$ and voltage generator, $V_{7}$, as shown in the right part of Fig. 15. Thereafter, the acquired disturbance signal is applied to the estimated $Z_{\text {out }_{C L}}(z)$ model. Then, an output voltage during the transient response is acquired. This result is known as the $Z_{\text {out }_{C L}}(z)$ estimation result. The simulation and estimated results are compared.

Two conditions must be met to ensure that the transient response is stable, that is, (a) the number of oscillations at the transient response does not exceed three and (b) the undershoot does not exceed the tolerance value given in the datasheet. 


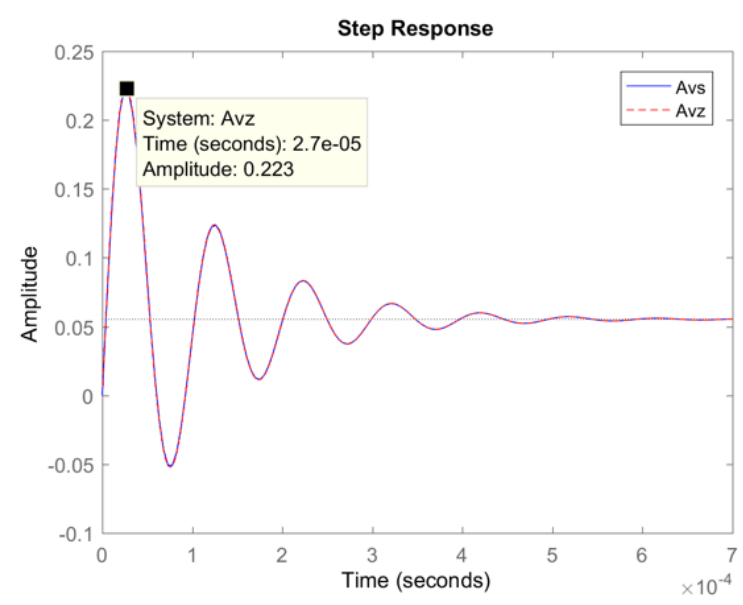

Fig. 16. Step Response with Rising Time of $27 \mu$ s.

\section{RESUlTS AND DisCUSSION}

\section{A. Validation of Input Signal}

As a multisine signal is selected as the input signal for the LR method, two important factors need to be considered: (a) the number of sinusoidal signals and (b) the values of the sinusoidal frequency. These two factors play an essential role in the accuracy of the estimated physical parameters. In sum, the more suitable the number of sinusoidal signals and the value of the sinusoidal frequency signal used are the more accurate the estimated physical parameters will be.

Firstly, the number of sinusoidal signals depends on the number of frequency components or the number of coefficients in the $A_{v}$ model. In this study, we have six frequency components, as shown in (11); thus, at least three sinusoidal signals are required. However, four sinusoidal signals are used to increase the level of accuracy.

Secondly, the value of the sinusoidal frequency signal depends on the step response rising time of the $A_{v}$ model. Hence, the selected sinusoidal frequencies must reach a maximum of $37 \mathrm{kHz}$. Fig. 16 depicts the step response of the $A_{v}$ model. Selecting a frequency beyond $100 \mathrm{kHz}$ is not recommended because when the frequency exceeds $100 \mathrm{kHz}$, the magnitude decreases and undergoes phase changes, as shown in Fig. 17. Thus, the stability of the LVR is affected.

\section{B. Voltage Gain Model Estimation Results}

This section discusses the voltage gain estimation results, $A_{v_{L R}}$ obtained via the LR method. In this study, the parameters that are easy to measure and obtain, such as $R_{1}, R_{2}, C_{\text {out }}$, and $r_{d s}$, are replaced by $64 \mathrm{k} \Omega, 36 \mathrm{k} \Omega, 10 \mu \mathrm{F}$, and $65 \Omega$, respectively. The results of this estimation are shown in (29). The coefficients of the $A_{v_{L R}}$ model are listed in Table I.

$$
A_{v_{L R}}(z)=\frac{0.006905+0.000117 z^{-1}-0.006794 z^{-2}}{1-1.978 z^{-1}+0.982 z^{-2}}
$$

Before the simultaneous equations are solved, the polarity for the coefficient must be noted. For example, the coefficient

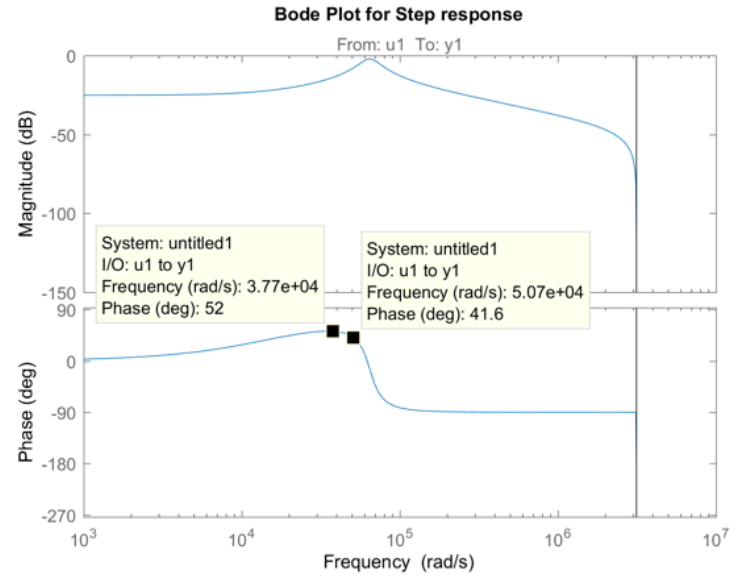

Fig. 17. Bode Plot with Frequencies of 37 dan $50 \mathrm{kHz}$.

TABLE I. Estimation of the Coefficients of Voltage Gain Model

\begin{tabular}{cc}
\hline Coefficient & Coefficient values \\
\hline$a_{1_{L R}}$ & 1.978000 \\
$a_{2}$ & -0.982000 \\
$b_{0 R}$ & 0.006905 \\
$b_{1_{L R}}$ & 0.000117 \\
$b_{2}$ & -0.006794 \\
\hline
\end{tabular}

for $a_{1_{L R}}$ is -1.978 based on (29), but it is incorrect because the polarity for the coefficient must be based on the equation of the LR method derived in (21). On the basis of (21), $a_{1_{L R}}$ and $a_{2_{L R}}$ have negative values. Therefore, the polarity for both coefficients must be inverted.

\section{Physical Parameter Estimation Results}

This section describes the results of the estimated internal physical parameters, i.e., $C_{g s} R_{o a} g_{m}, C_{g s} R_{o a}, g_{m}$, and $G_{e a} g_{m}$. Before the physical parameter estimation process can be performed, the values of the physical parameters must be set randomly. Four examples of parameter sets are provided for the parameter estimation process, as shown in Table II, and the following equation is used to find the accuracy of the estimated physical parameters:

$$
\text { Accuracy }=\left|\frac{T P P-E P P}{T P P}\right| \times 100 \%
$$

where TPP and EPP are target physical parameter and estimated physical parameter, respectively.

As shown in Table II, the results of the estimated physical parameters indicate that the MBA is capable of estimating the physical parameters of the LVR circuit with a high level of accuracy of approximately $90 \%$. However, the estimation results of these parameters are obtained on the basis of $1 \mu \mathrm{s}$ sampling time and multisine input signal with $5,10,15$, and $20 \mathrm{kHz}$ frequencies. 
TABLE II. Four EXAMPLES OF PHySicAl PARAMETERS OF THE LVR CIRCUIT

\begin{tabular}{ccccc}
\hline Set & Parameters & EPP & TPP & Accuracy (\%) \\
\hline & $C_{g s} R_{\text {oa }} g_{m}$ & $7.3865 \times 10^{-6}$ & $7.3800 \times 10^{-6}$ & 99.91 \\
$\mathrm{~A}$ & $C_{g s} R_{\text {oa }}$ & $6.0088 \times 10^{-5}$ & $6.0000 \times 10^{-5}$ & 99.85 \\
& $G_{\text {ea }} g_{m}$ & 6.7011 & 6.9126 & 96.94 \\
& $g_{m}$ & $127.00 \times 10^{-3}$ & $123.00 \times 10^{-3}$ & 96.75 \\
& & & & \\
& $C_{g s} R_{\text {oa }} g_{m}$ & $7.3900 \times 10^{-6}$ & $7.5000 \times 10^{-6}$ & 98.53 \\
$\mathrm{~B}$ & $C_{g s} R_{\text {oa }}$ & $6.0456 \times 10^{-5}$ & $6.0500 \times 10^{-5}$ & 99.93 \\
& $G_{\text {ea }} g_{m}$ & 6.9119 & 6.9595 & 99.32 \\
& $g_{m}$ & $128.90 \times 10^{-3}$ & $123.50 \times 10^{-3}$ & 95.63 \\
& & & & \\
& $C_{g s} R_{\text {oa }} g_{m}$ & $7.4410 \times 10^{-6}$ & $7.6200 \times 10^{-6}$ & 97.65 \\
$\mathrm{C}$ & $C_{g s} R_{\text {oa }}$ & $6.0829 \times 10^{-5}$ & $6.1000 \times 10^{-5}$ & 99.72 \\
& $G_{\text {ea }} g_{m}$ & 7.1253 & 6.8282 & 95.64 \\
& $g_{m}$ & $133.90 \times 10^{-3}$ & $122.50 \times 10^{-3}$ & 90.69 \\
& & & & \\
& $C_{g s} R_{\text {oa }} g_{m}$ & $7.2637 \times 10^{-6}$ & $7.2699 \times 10^{-6}$ & 99.91 \\
$\mathrm{D}$ & $C_{g s} R_{\text {oa }}$ & $5.9304 \times 10^{-5}$ & $5.9400 \times 10^{-5}$ & 99.83 \\
& $G_{\text {ea }} g_{m}$ & 6.2870 & 6.8436 & 91.87 \\
& $g_{m}$ & $114.20 \times 10^{-3}$ & $122.38 \times 10^{-3}$ & 93.32 \\
\hline & & & &
\end{tabular}

\section{Output Impedance Model Estimation Results}

This section explains the estimated output impedance model, $Z_{\text {out }_{C L}}(z)$, a comparison between the $Z_{\text {out }}$ CL $(z)$ simulation results and the $Z_{\text {out }}(z)$ estimated results, and the transient response for different ESR values. Firstly, the estimated output impedance model is discussed. After the physical parameters are estimated, the values of the set A parameters shown in Table II are substituted into the estimated $Z_{\text {out }}(z)$ model. Finally, the transfer function of the $Z_{\text {out }_{C L}}(z)$ model is generated, as shown in (31).

$$
\begin{aligned}
& \left(0.6521 z^{7}-2.276 z^{6}+2.336 z^{5}\right. \\
& +0.474 z^{4}-2.5 z^{3}+1.756 z^{2} \\
& Z_{\text {out }_{C L}}(z)=\frac{-0.4876 z+0.0478)}{\left(1.013 z^{7}-4.84 z^{6}+9.458 z^{5}\right.} \\
& -9.782 z^{4}+5.718 z^{3}-1.876 z^{2} \\
& +0.3204 z-0.0221)
\end{aligned}
$$

Secondly, a comparison of the simulation and estimation results of the $Z_{\text {out }}$ CL $(z)$ model is displayed in Fig. 18, where the output current and ESR are set to $115 \mathrm{~mA}$ and $2 \Omega$, respectively.

Fig. 18 shows that the two results are nearly the same. This similarity is due to the accurate estimated physical parameters, as shown in Table II. In sum, the result of the estimated $Z_{\text {out }_{C L}}(z)$ model is validated by the simulation of the LVR circuit with OrCAD Capture CIS software. Fig. 19 shows the input voltage of the input signal, while Fig. 20 shows the output voltage as the output signal.

Finally, the analysis of LVR stability is conducted on the basis of the estimated $Z_{\text {out }}$ CL $(z)$ model, as shown in (31). In this study, the following conditions should be met to ensure the stable level of the LVR circuit: (a) the number of oscillations does not exceed three oscillations, and (b) the undershoot does not exceed the tolerance value as provided in the datasheet,

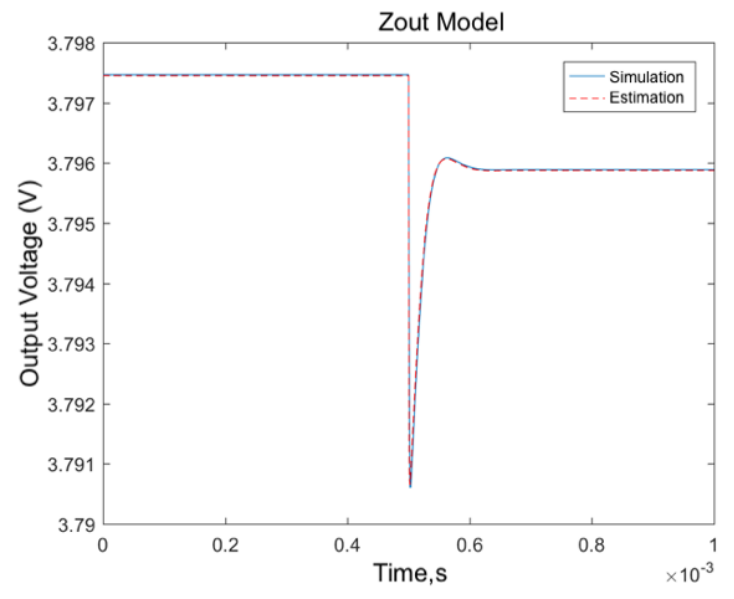

Fig. 18. Comparison of Simulation and Estimation of $Z_{o u t} C L(z)$ Model.

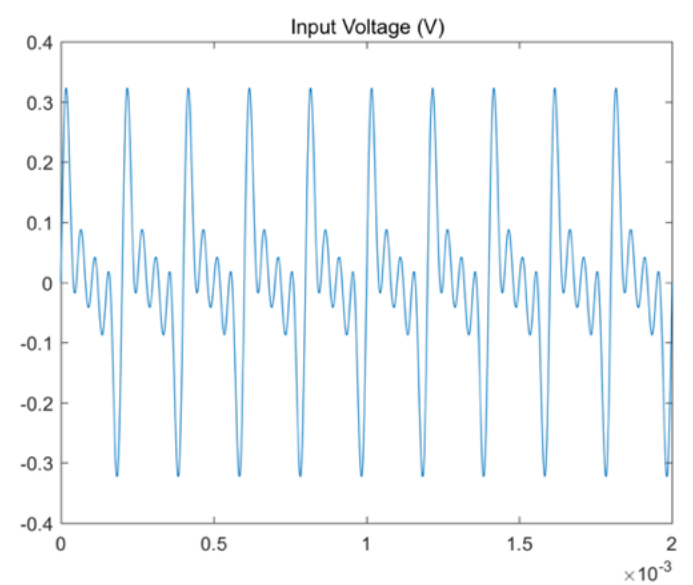

Fig. 19. Multisine as the Input Signal for the $A_{v_{L R}}$ Model of LVR Circuit.

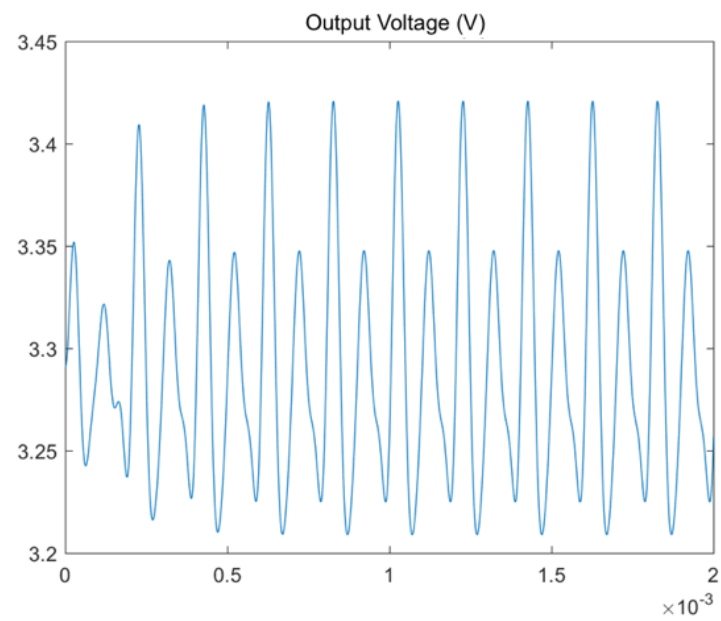

Fig. 20. Output Signal for the $A_{v_{L R}}$ Model LVR Circuit.

which is $20 \mathrm{mV}$. Fig. 21 to 23 illustrate the transient response results along with the different ESR values given an output current is set at $115 \mathrm{~mA}$. 


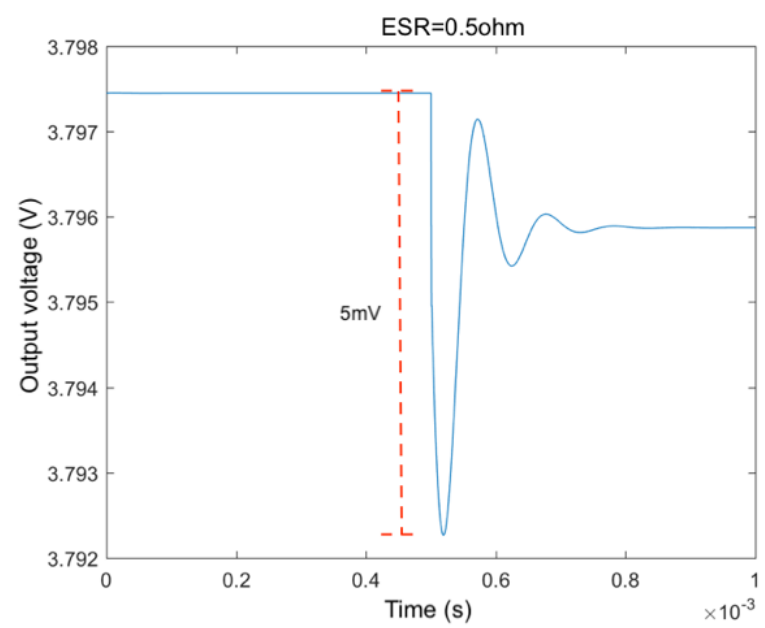

Fig. 21. Transient Response of Estimated $Z_{\text {out } C L}(z)$ with $\mathrm{ESR}=0.5 \Omega$.

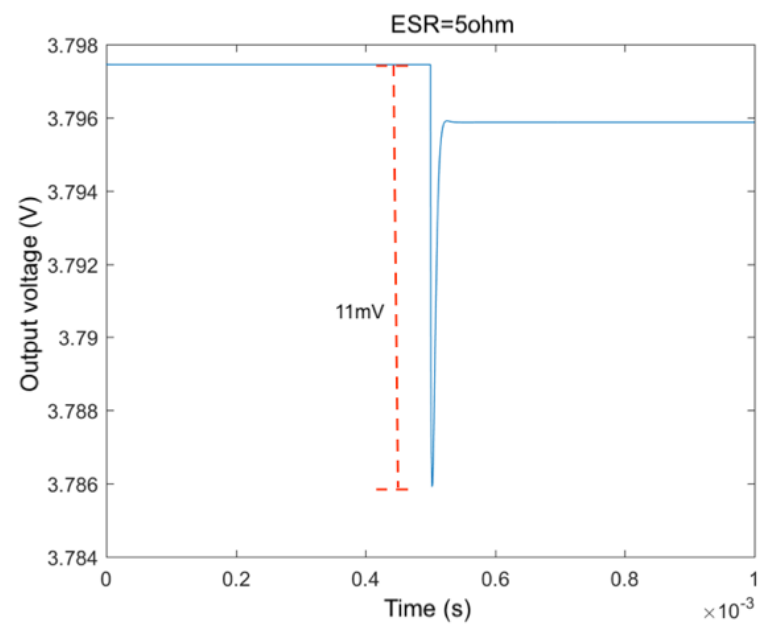

Fig. 22. Transient Response of Estimated $Z_{\text {out } C L}(z)$ with $\operatorname{ESR}=5 \Omega$.

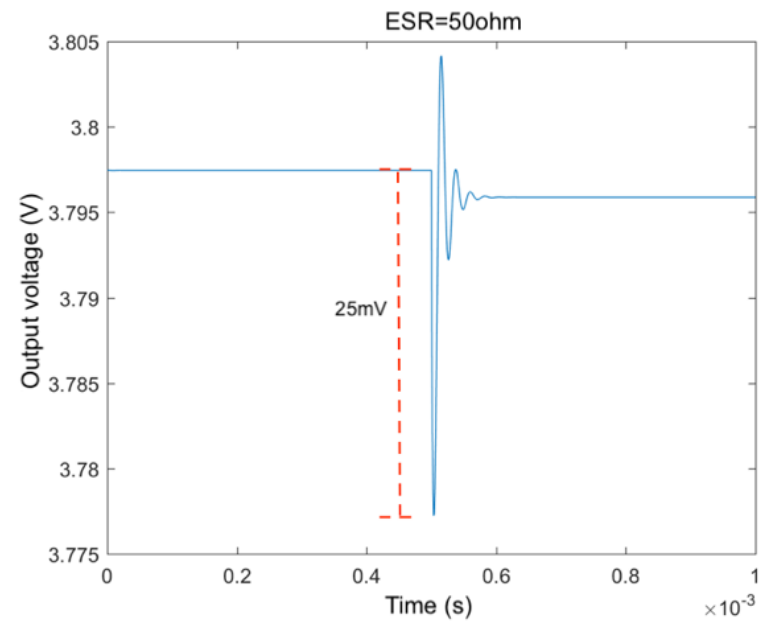

Fig. 23. Transient Response of Estimated $Z_{o u t} C L(z)$ with ESR $=50 \Omega$.

Fig. 21 illustrates the results of a transient response with an ESR of $0.5 \Omega$. Although the results reflect a small undershoot, the relatively high number of oscillations causes the LVR to operate in unstable conditions. Figure 22 shows a stable LVR with ESR of $5 \Omega$. This result is explained as follows: the transient response contains less than three oscillations, and the undershoot is in the appropriate range. Fig. 23 illustrates the results of a transient response with an ESR of $50 \Omega$. Although the number of oscillations does not exceed three oscillations, and the undershoot exceeds its tolerance value, the LVR fails to function correctly. In sum, an excessively low ESR causes oscillation to occur, whereas a high ESR produces a large undershoot. These two conditions result in an unstable output voltage.

\section{CONCLUSION}

In conclusion, this study was successfully conducted, and the results obtained prove that the objectives were achieved. Firstly, this study successfully developed a method for estimating the physical parameters of LVR circuits through the MBA. Secondly, the results of the physical parameter estimation were successfully obtained with a high level of accuracy. Finally, LVR stability was successfully studied and analyzed through a transient response. Although the objectives of this study were achieved, the proposed MBA method may still be improved further. For example, study can be implemented using real LVR circuits to strengthen the concept of the MBA practically and validate the results obtained.

Herein, the MBA method is highly dependent on the LVR circuit supplied by the manufacturer, and this study can only be conducted when the LVR circuit is given in the datasheet. Therefore, this study can be improved in the future to solve the problem with the use of machine learning. For example, machine learning can analyze and provide some examples of possible LVR circuit sets through algorithmic learning and computing models.

\section{ACKNOWLEDGMENT}

The authors would like to thank Ministry of Higher Education Malaysia for the financial support under grant number FRGS/1/2019/TK04/UKM/03/1.

\section{REFERENCES}

[1] C. Wilson, T. Hargreaves, and R. Hauxwell-Baldwin, "Benefits and risks of smart home technologies," Energy Policy, vol. 103, pp. 72-83, 2017.

[2] Y. Shi, A. Wang, J. Cao, and Z. Zhou, "A transient-enhanced voltage regulator with stability and power-supply-rejection boosting," Nanoscale Research Letters, vol. 14, no. 368, pp. 1-11, 2019.

[3] J. Wang, B. Bao, J. Xu, G. Zhou, and W. Hu, "Dynamical effects of equivalent series resistance of output capacitor in constant on-time controlled buck converter," IEEE Transactions on Industrial Electronics, vol. 60, no. 5, pp. 1759-1768, 2013.

[4] C. K. Chava, and J. Silva-Martínez, "A frequency compensation scheme for LDO voltage regulators," IEEE Transactions on Circuits and Systems I: Regular Papers, vol. 51, no. 6, pp. 1041-1050, 2004.

[5] M. Day, "Understanding low drop out (LDO) regulators," in 2006 Texas Instruments Portable Power Design Seminar, pp. 1-7, 2006.

[6] J. Falin, and J. Cummings, "ESR, stability, and the LDO regulator," Texas Instruments Application Report, pp. 1-6, 2020.

[7] B. S. Lee, "Technical review of low dropout voltage regulator operation and performance," Texas Instruments Application Report, pp. 1-25, 1999.

[8] E. Rogers, "Stability analysis of low-dropout linear regulators with a PMOS pass element," Power Management, pp. 10-13, 1999. 
[9] Rohm Semiconductor, "Simple test method for estimating the stability of linear regulators," Application Note, pp. 1-4, 2016.

[10] A. H. Musa, M. H. M. Zaman, R. Mohamed, and M. M. Mustafa, "Characterization of voltage regulators by automated equivalent series resistance," in Proceedings of the 2014 IEEE Conference on System, Process and Control (ICSPC 2014), pp. 68-72, 2014.

[11] S. Zamri, M. H. M. Zaman, M. F. Ibrahim, A. A. Ibrahim, and M. M. Mustafa, "Noninvasive stability measurement of linear voltage regulator in the closed-loop condition," International Journal of Advanced Trends in Computer Science and Engineering, vol. 10, no. 1.6, pp. 167-172, 2019.

[12] M. H. M. Zaman, M. M. Mustafa, and A. Hussain, "Estimation of voltage regulator stable region using radial basis function neural network," Journal of Telecommunication, Electronic and Computer Engineering, vol. 10, no. 2-8, pp. 63-66, 2018.

[13] M. H. M. Zaman, M. M. Mustafa, M. A. Hannan, and A. Hussain, "Neural network based prediction of stable equivalent series resistance in voltage regulator characterization," Bulletin of Electrical Engineering and Informatics, vol. 7, no. 1, pp. 134-142, 2018.

[14] Z. Guo, and J. Savir, "Analog circuit test using transfer function coefficient estimates," in Proceedings of the International Test Conference, pp. 1155-1163, 2003.

[15] Z. Guo, and J. Savir, "Coefficient-based test of parametric faults in ana$\log$ circuits," IEEE Transactions on Instrumentation and Measurement, vol. 55, no. 1, pp. 150-157, 2006.

[16] A. Kavithamani, V. Manikandan, and N. Devarajan, "Soft fault diagnosis of analog circuit using transfer function coefficients," in Proceedings of the International Conference on Process Automation, Control and Computing, pp. 1-6, 2011.

[17] S. Sindia, V. D. Agrawal, and V. Singh, "Non-linear analog circuit test and diagnosis under process variation using V-Transform coefficients,' in Proceedings of the IEEE VLSI Test Symposium, pp. 64-69, 2011.
[18] V. Valdivia, A. Barrado, A. Lazaro, P. Zumel, and C. Raga, "Easy modeling and identification procedure for "black box" behavioral models of power electronics converters with reduced order based on transient response analysis," in Proceedings of the IEEE Applied Power Electronics Conference and Exposition, pp. 318-324, 2009.

[19] V. Valdivia, A. Barrado, A. M. Roldan, C. Fernandez, and P. Zumel, "Black box modeling of DC-DC converters based on transient response analysis and parametric identification methods," in Proceedings of the 25th Annual IEEE Applied Power Electronics Conference and Exposition, pp. 1131-1138, 2010.

[20] V. Valdivia, A. Lazaro, A. Barrado, P. Zumel, C. Fernandez, and M. Sanz, "Black box modeling of three-phase voltage source inverters for system-level analysis," IEEE Transactions on Industrial Electronics, vol.59, no. 9, pp. 3648-3662, 2012.

[21] C. C. Bilberry, M. S. Mazzola, and J. Gafford, "Power supply on chip (PwrSoC) model identification using black-box modeling techniques," in Proceedings of the 27th Annual IEEE Applied Power Electronics Conference and Exposition, pp. 1821-1825, 2012.

[22] H. Luo, Y. Wang, H. Lin, and Y. Jiang, "Module level fault diagnosis for analog circuits based on system identification and genetic algorithm," Measurement, vol. 45, no. 4, pp. 769-777, 2012.

[23] Y. Yusof, N. Abd. Rahim, "Functional simulation model for single phase pulse width modulation-voltage source inverter (PWM-VSI) using switching function concept," Jurnal Kejuruteraan, vol. 23, pp. 49-56, 2011.

[24] C. Simpson, "Linear and switching voltage regulator fundamental part 1,” Texas Instruments, pp. 1-30, 2011.

[25] S. Okiy, C. Chukwuemeka Nwobi-Okoye, and A. Clement Igboanugo, "Transfer function modelling: A literature survey," Research Journal of Applied Sciences, Engineering and Technology, vol. 11, no. 11, pp. $1265-1279,2015$.

[26] M. H. M. Zaman, M. M. Mustafa, and A. Hussain, "Black-box modeling of low dropout voltage regulator based on two-port network parameter identification," in Proceedings of the 2015 IEEE 11th International Colloquium on Signal Processing and Its Applications, pp. 78-83, 2015. 